\title{
Extracorporeal membrane oxygenation
}

\author{
Warwick Butt ${ }^{1-3}$ and Graeme MacLaren ${ }^{1,2,4 *}$
}

Addresses: ${ }^{1}$ Paediatric Intensive Care Unit, Royal Children's Hospital, 50 Flemington Road, VIC 3052, Australia; ${ }^{2}$ Department of Paediatrics, The University of Melbourne, VIC 3010, Australia; ${ }^{3}$ Murdoch Children's Research Institute, Clinical Sciences, 50 Flemington Road, VIC 3052 , Australia; ${ }^{4}$ Cardiothoracic Intensive Care Unit, National University Health System, 5 Lower Kent Ridge Road, Singapore 119074

* Corresponding author: Graeme MacLaren (gmaclaren@iinet.net.au)

FI000Prime Reports 2013, 5:55 (doi:10.12703/P5-55)

This is an open-access article distributed under the terms of the Creative Commons Attribution-Non Commercial License (http://creativecommons.org/licenses/by-nc/3.0/legalcode), which permits unrestricted use, distribution, and reproduction in any medium, provided the original work is properly cited. You may not use this work for commercial purposes.

The electronic version of this article is the complete one and can be found at: http://f1000.com/prime/reports/m/5/55

\begin{abstract}
Extracorporeal membrane oxygenation (ECMO) is an advanced form of life support technology whereby venous blood is oxygenated outside of the body and returned to the patient. ECMO was initially used as last-resort rescue therapy for patients with severe respiratory failure. Over the last four decades, it has developed into a safe, standard therapy for newborns with progressive cardiorespiratory failure, as a resuscitation therapy after cardiac arrest, and in combination with other treatments such as hypothermia and various blood filtration therapies. ECMO has also become routine for children and adults with all forms of cardiogenic shock and is also routine in early graft failure after transplantation. The one area of ongoing debate is the role of ECMO in adults with hypoxemic respiratory failure. As ECMO equipment becomes safer, earlier use improves patient outcomes. Several modifications of the two basic venovenous and venoarterial ECMO systems are now occurring, as are many minor variations in cannulation strategies and systems of care for patients receiving ECMO. The indications and situations in which ECMO have been tried continue to change, and ECMO for sub-acute and chronic illnesses is now commonplace, as is the use of ECMO in patients with clinical problems previously regarded as contraindications, such as sepsis, malignancy, and immunosuppression.
\end{abstract}

\section{Introduction}

ECMO is an advanced form of life support technology, whereby venous blood is removed from the patient and passed through a membrane lung where oxygen is added and carbon dioxide removed. The blood is then returned to the patient [1]. Both circulatory and respiratory support may be provided if oxygenated blood is returned to an artery (venoarterial ECMO). If oxygenated blood is returned to a vein (venovenous ECMO), then only respiratory support is provided. ECMO can keep patients alive for days to weeks even when the heart or lungs are completely non-functional [2]. Other forms of extracorporeal technology can be added onto the ECMO circuit to provide support for other organ systems [3]. For example, connecting a hemofiltration circuit to the ECMO system to serve as an artificial kidney is frequently done in many centers.
ECMO began in the early 1970 s as a prolonged form of cardiopulmonary bypass to treat severe respiratory failure. The initial approach with ECMO was to siphon blood from a vein and return it to an artery (venoarterial ECMO), and the duration was fixed at 5 days. Treatment was started when death was viewed as near-certain, because of concerns about the severity of complications at the time. The first randomized controlled trial [4], published in 1979, reflected this, and patient outcomes were poor in both conventional and ECMO groups. One of the authors (Robert Bartlett) decided that the therapy held promise but what was required were patients with a disease that would recover quickly within 5 days. He successfully applied ECMO in newborn infants with acute respiratory failure and persistent pulmonary hypertension of the newborn, which usually resolves within a few days of birth. 
During the 1980s, the use of ECMO rapidly proliferated as early successes with newborn respiratory failure were reported. Trials by committed clinicians used novel statistical methods to try to establish the benefits of ECMO, but controversy remained as to the efficacy and safety of the technology. A randomized trial of inverse ratio ventilation and extracorporeal carbon dioxide removal in adult respiratory distress syndrome [5] showed no benefit, further dissuading clinicians in adult medicine from adopting the technology. However, ECMO was used in children with cardiorespiratory failure and sepsis and adapted to children with cardiogenic shock following cardiac surgery. As results improved, ECMO gradually gained credibility as a useful treatment in children, and a randomized controlled trial of ECMO in severe neonatal respiratory failure, published in 1996, showed improved survival and better long-term neurodevelopmental outcomes in the ECMO group $[6,7]$.

Refinements in clinical management and ECMO equipment continued, and ECMO gradually changed from a "rescue therapy of last resort" to a standard therapy with clear indications and contraindications. Results continued to improve, and innovations in circuit design resulted in wider applications to more diverse clinical situations. The term ECMO was changed to extracorporeal life support (ECLS) and this acronym is used instead of ECMO in many parts of the world. Resuscitation and transport of critically ill patients on ECMO has become routine. There has also been increasing use of associated adjunctive therapies, such as hypothermia or various types of filtration therapies, as part of the ECMO circuit. The structure and nature of ECMO programs and models of care for patients on ECMO are also varied and depend on local skills and expertise rather than any one definitive or "best practice" model. This leads to variations in practice in different parts of the world.

\section{Goals of support}

The fundamental goal of ECMO is to enhance systemic oxygen delivery. The oxygen saturation goals vary from patient to patient and from center to center. The circuit flow rate chosen may also vary substantially and consequently alters much of how ECMO is done. For example, in pediatric sepsis guidelines, a maximum blood flow rate of $110 \mathrm{~mL} / \mathrm{kg} / \mathrm{min}$ has been recommended [8], although there are reports of improved survival with 150 to $200 \mathrm{~mL} / \mathrm{kg} / \mathrm{min}$ [9]. For adult ECMO patients with acute respiratory distress syndrome, some centers aim for an arterial oxygen saturation of more than $80 \%$, while others prefer more than $90 \%$. These differences in management goals have an impact on the way ECMO is conducted, with regards to catheter size and cannulation strategies.

\section{Cannulation strategies and organ support}

The type of ECMO applied is determined by the type of organ support required, the type of patient being treated, and the desired outcome. Support of respiratory function is usually done with venovenous cannulation; oxygen delivery is determined by the proportion of the patient's cardiac output being diverted through the extracorporeal circuit (i.e. circuit flow rate), whilst carbon dioxide clearance is principally determined by the amount of fresh gas flow through the oxygenator. If there is a large amount of blood flow required for the circuit or if the ECMO catheters are in close proximity to each other, then recirculation is possible. This is the term for the recycling of already oxygenated blood back into the circuit, with a consequent decrease in overall patient oxygen delivery. Patient diagnoses (acute or chronic disease), mobility and need for physiotherapy (e.g. chronic obstructive pulmonary disease, cystic fibrosis), and ultimate destination (recovery or transplantation) all subtly affect management strategies. Thus many different venovenous strategies exist, depending on the goals of respiratory support. These include single-catheter tidal flow, double-lumen systems, or two- and three-catheter systems.

Support of cardiac function (either single or both ventricles) will require venoarterial cannulation, and the degree of support is determined by the amount of circuit blood flow. Blood may or may not be ejected by the failing ventricle. The diagnosis, age of the patient, and the ultimate destination are important in determining the type of cannulation performed. In infants who weigh less than $10 \mathrm{~kg}$, these catheters are generally placed in the neck, and in the groin in older children and adults. After cardiac surgery, some centers use central or transthoracic catheter placement.

\section{Complications of different cannulation strategies}

Catheter placement is either by surgical cut-down and insertion under vision or percutaneous with the use of ultrasound. In small infants and children, surgery is generally preferred, and vessel repair is performed at removal; in adults, percutaneous placement is standard, with surgery occasionally needed to help with difficult access or to repair arterial vessels.

Central cannulation requires the surgical insertion of catheters into major vessels or directly into either atria and therefore carries an increased risk of bleeding and infection. The sternum and skin may or may not be closed, and further major surgery is required to remove these catheters. This strategy is common in small infants but increasingly uncommon in adults.

Venoarterial ECMO in the groin vessels can be associated with cerebral hypoxemia if ventricular function recovers 
whilst severe respiratory failure is present [10]. The right ventricle propels blood through the poorly functioning lungs, which leads to desaturated blood coming into the left ventricle and then being ejected into the ascending aorta. This is known as differential cyanosis. It can be treated with a number of different strategies, including increasing ventilatory support of the lungs (by increasing the fraction of inspired oxygen or increasing the amount of positive end-expiratory pressure), adding an additional venous catheter to return a proportion of oxygenated blood into the venous system (venoarteriovenous ECMO), moving the arterial return catheter more proximally into the right subclavian or carotid artery, or changing to central ECMO. Femoral cannulation is associated with substantial vascular complications in $10 \%$ of cases [9] and these may include ischemia and the need for amputation.

\section{Complications of extracorporeal membrane oxygenation}

The complications of all extracorporeal technology are similar and include bleeding, thromboembolism, access difficulties, and infection. Mechanical equipment failure is now very uncommon with modern systems. The incidence of major complications that impact on long-term outcomes is also lower and varies depending on the type of patient, severity of illness, and indication for ECMO. As indications for ECMO and treatment protocols change, so do the complication rates. Use during cardiac arrest increases the likelihood of cerebral hemorrhage; use after trauma, in severe multiorgan failure, or initiation after seven days of mechanical ventilation is complicated and more frequently associated with bleeding.

\section{Circuit design and equipment}

The basic ECMO circuit is shown in Figure 1. The circuit consists of vascular access catheters, a pump, an oxygenator, a temperature control system, monitors, and access points.

Many different catheters can be employed, but catheter selection is based on local preference and little debate occurs about these. However, much debate exists about the relative merits of the roller pump (North America) and the centrifugal pump (Europe, Asia, and Australasia). Both pumps have potential problems. The roller pump uses gravity drainage and a bladder as a reservoir and thus clotting is possible. Tubing rupture has also occurred due to compression. The centrifugal pump generates suction at the inlet, and hemolysis is possible. A recent study highlighted this as a potentially serious issue for newborn infants [11].

A number of different oxygenators are used worldwide. Heparin bonding and newer materials are used to minimize
Figure I. Basic ECMO circuit

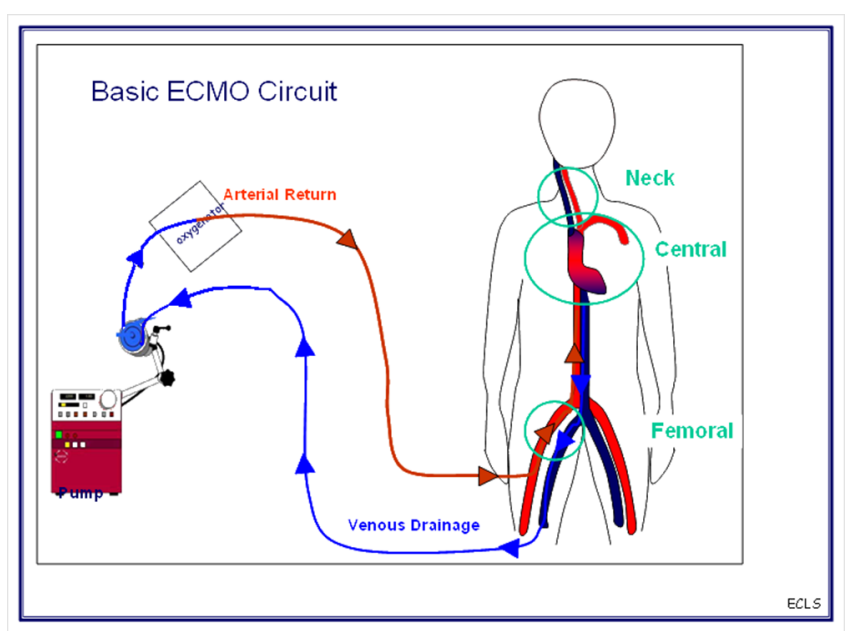

Venous blood is removed from the patient and pumped through an oxygenator where oxygen enters into the blood and carbon dioxide diffuses out. The oxygenated blood is then pumped back into an artery or vein. Venous and arterial access is obtained from vessels in the groin, neck or chest.

the inflammatory response and platelet adhesiveness to these materials. It is likely that a truly biocompatible plastic will eventually be developed (possibly with an endothelial coating) and obviate the need for anticoagulation. The different oxygenators have different resistances and gas exchange properties, but in practice these do not substantially alter patient outcome and thus local preferences determine which is used.

In-line saturation monitors are being replaced by noncontact external devices that monitor temperature, blood saturation, and flow. Standards for the use of pressure monitors vary, but those pediatric programs that use centrifugal pumps tend to monitor access and return pressures, while programs that use roller pumps tend to monitor pre- and post-oxygenator pressures. This latter approach is common in adult centers irrespective of the type of pump used.

Access points to the circuit have obvious benefits for circuit sampling, monitoring, and connection of other devices (such as dialysis) but these are also points of turbulent blood flow and potential thrombus formation. Circuit bridges (connections between access and return parts of the circuit adjacent to the cannulas) are points of stasis and blood clotting. These were useful to allow circuit changes with minimal disruption of patient support; with modern equipment, many pediatric centers and most adult centers are increasingly eliminating these from the ECMO circuit. 


\section{Anticoagulation}

Anticoagulation is essential for the safe use of ECMO at present. A number of key factors influence the likelihood of blood clots forming in the circuit, in particular the patient's inherent coagulability and the rate of circuit blood flow. Newborn infants, patients with a large systemic inflammatory response after cardiopulmonary bypass, or patients with disseminated intravascular coagulation from sepsis are likely to have circulating pro-coagulants and develop clots within a circuit. Stasis of blood will also lead to clots and therefore low circuit blood flow rates require increased anticoagulation. The commonest anticoagulant is heparin, but enoxaparin, bivilirudin, argatroban, and antiplatelet agents such as prostacycline have been used.

Institutions all use different protocols of anticoagulation and monitor anticoagulation in different ways. Blood tests of clotting effectiveness, such as activated partial thromoboplastin time, prothrombin time, anti-Xa levels, activated clotting time, and thromboelastography, have all been used to assess the balance between bleeding and thrombosis. Pediatric programs tend to initiate anticoagulation substantially earlier and aim for higher targets than adult programs because of the lower blood flow rates, larger circuit volumes relative to patient blood volume, and differences in infant coagulation physiology.

\section{Indications and contraindications}

The indications for ECMO are continually changing. Initial indications were acute reversible heart or lung disease in patients likely to die. However, high doses of inotropes and high pressure/volume ventilation are dangerous; prolonged hypotension or hypoxia also leads to multiorgan failure. Coupled with the increasing safety of ECMO, this realization has led to the earlier use of ECMO with consequent patient benefits.

ECMO has now become established as a means of resuscitating patients from acute cardiogenic shock, cardiac arrest, and acute fulminant respiratory failure; this gives time to identify other treatments that may be effective. Bridging to long-term mechanical support (such as ventricular assist devices) and transplantation is routine [12-14]. The role of ECMO in adults with severe respiratory failure continues to be debated, but increasing numbers of patients are receiving the treatment. Much of the debate revolves around timing and therapeutic strategies rather than whether it works [15-19].

ECMO was historically contraindicated in patients with sepsis and immunosuppression, but now survival of these patients can be as high as $50 \%$ to $75 \%$. Similarly, patients with trauma or bleeding were not offered ECMO, but there are now reports of successful outcomes in these patients.

\section{Outcomes}

Survival after ECMO is determined by many factors, including the nature and severity of disease, age, size, preexisting patient co-morbidities, and attitudes towards quality of life after critical illness. These factors are independent of any ECMO complications; any complications that affect patient outcome such as cerebral hemorrhage or systemic thromboembolism are additive.

\section{Evidence of benefit and current role of extracorporeal membrane oxygenation}

There are no trials that demonstrate the absolute benefit of ECMO in improving survival or quality of life. One reason for the paucity of controlled trials is that it is extremely difficult to design a randomized controlled study of life support that is ethically acceptable and yet has the utmost scientific rigor $[20,21]$. There can be no question that further trials of ECMO are needed, but controversy exists as to how these should be conducted [22]. Nonetheless, over 55,000 patients have been reported with a survival to discharge of more than $60 \%$ [23], with outcomes varying with age and diagnosis.

Sufficient experience with ECMO has accumulated worldwide that it is regarded as a standard of care for cardiogenic shock in both pediatric and adult medicine $[24,25]$. It is also regarded as a standard of care in refractory neonatal and pediatric respiratory failure [6]. However, the debate continues over adult respiratory failure in many centers [19]. Two recent reports illustrated the difficulties of studying ECMO for this indication and both involved adults with hypoxic respiratory failure from influenza A (H1N1). The first study used a number of different statistical techniques, including propensity matching to compare two similar groups of patients in the United Kingdom; one group received ECMO and the other did not [26]. Transfer to an ECMO-capable hospital was associated with lower mortality irrespective of the statistical technique used. The second study used similar statistical methods to analyze a comparable French cohort but was unable to demonstrate that ECMO was associated with improved survival [27]. However, 51 patients in this series received ECMO but were not able to be matched to non-ECMO controls. Despite having significantly worse gas exchange before ECMO than the matched cohort, they had significantly lower mortality ( $22 \%$ vs. $50 \%, P<0.01)$. The presence of this group made interpretation of the study's results more difficult. Although a large randomized controlled trial showed that referral to an ECMOcapable center was associated with reduced mortality [28], the methodology was heavily criticized. Complex and potentially conflicting results such as these continue to fuel the controversy. Another multinational trial of 
ECMO in this patient group is under way (ClinicalTrials. gov identifier: NCT01470703).

\section{Extracorporeal Life Support Organization}

The Extracorporeal Life Support Organization (ELSO) was founded in 1989. The group maintains an international registry that contained details of 55,668 patients by July 2013. The results of this registry are published biannually and distributed to members. The complications in patients who receive ECMO are recorded by ELSO; this list is very comprehensive and includes all events whether due to ECMO or not.

\section{Conclusions}

ECMO is a standard therapy in critical care. It is used as a treatment for acute severe cardiorespiratory failure and as a resuscitation strategy in many clinical scenarios. It is also used as a "bridge" to other treatments and transplantation. It continues to be applied to more complex and chronic situations. It is being integrated into multiple-organ support therapies. Substantial improvements in biotechnology and clinical practices over the last 40 years have allowed ECMO to provide a vital role in acute organ support in patients of all ages. It is likely that further such advances will diminish complication rates, facilitate more widespread adoption of the technology in middle- and high-income countries, and improve outcomes from refractory heart, lung, and multiorgan failure.

\section{Abbreviations}

ECMO, extracorporeal membrane oxygenation; ELSO, Extracorporeal Life Support Organization.

\section{Disclosures}

The authors declare that they have no disclosures.

\section{References}

I. Chauhan S, Subin S: Extracorporeal membrane oxygenation, an anesthesiologist's perspective: physiology and principles. Part I. Ann Card Anaesth 201 I, 14:218-29.

2. Sidebotham D, McGeorge A, McGuinness S, Edwards M, Willcox T, Beca J: Extracorporeal membrane oxygenation for treating severe cardiac and respiratory failure in adults: part 2-technical considerations. J Cardiothorac Vasc Anesth 2010, 24:164-72.

\section{FlOOPrime}

\section{RECOMMENDED}

3. Hirshberg E, Miller RR, Morris AH: Extracorporeal membrane oxygenation in adults with acute respiratory distress syndrome. Curr Opin Crit Care 2013, 19:38-43.

4. Zapol WM, Snider MT, Hill JD, Fallat RJ, Bartlett RH, Edmunds LH, Morris AH, Peirce EC, Thomas AN, Proctor HJ, Drinker PA, Pratt PC, Bagniewski A, Miller RG: Extracorporeal membrane oxygenation in severe acute respiratory failure. A randomized prospective study. JAMA 1979, 242:2193-6.

5. Morris AH, Wallace CJ, Menlove RL, Clemmer TP, Orme JF, Weaver LK, Dean NC, Thomas F, East TD, Pace NL, Suchyta MR, Beck E, Bombino M, Sittig DF, Böhm S, Hoffmann B, Becks H, Butler S, Pearl J, Rasmusson B: Randomized clinical trial of pressure-controlled inverse ratio ventilation and extracorporeal $\mathrm{CO} 2$ removal for adult respiratory distress syndrome. Am J Respir Crit Care Med I 994, I 49:295-305.

6. UK collaborative randomised trial of neonatal extracorporeal membrane oxygenation. UK Collaborative ECMO Trail Group. Lancet 1996, 348:75-82.

7. McNally H, Bennett CC, Elbourne D, Field DJ: United Kingdom collaborative randomized trial of neonatal extracorporeal membrane oxygenation: follow-up to age 7 years. Pediatrics 2006, I I 7:e845-54.

8. Brierley J, Carcillo JA, Choong K, Cornell T, Decaen A, Deymann A, Doctor A, Davis A, Duff J, Dugas M, Duncan A, Evans B, Feldman J, Felmet K, Fisher G, Frankel L, Jeffries H, Greenwald B, Gutierrez J, Hall M, Han YY, Hanson J, Hazelzet J, Hernan L, Kiff J, Kissoon N, Kon A, Irazuzta J, Irazusta J, Lin J, et al.: Clinical practice parameters for hemodynamic support of pediatric and neonatal septic shock: 2007 update from the American College of Critical Care Medicine. Crit Care Med 2009, 37:666-88.

9. MacLaren G, Butt W, Best D, Donath S: Central extracorporeal membrane oxygenation for refractory pediatric septic shock. Pediatr Crit Care Med 201 I, I 2:133-6.

\section{FIOOOPrime}

\section{RECOMMENDED}

10. Bisdas T, Beutel G, Warnecke G, Hoeper MM, Kuehn C, Haverich A, Teebken OE: Vascular complications in patients undergoing femoral cannulation for extracorporeal membrane oxygenation support. Ann Thorac Surg 201 I, 92:626-3I.

\section{FIOOOPrime
RECOMMENDED}

II. Barrett CS, Jaggers J], Cook EF, Graham DA, Rajagopal SK, Almond CS, Seeger JD, Rycus PT, Thiagarajan RR: Outcomes of neonates undergoing extracorporeal membrane oxygenation support using centrifugal versus roller blood pumps. Ann Thorac Surg 20|2, 94:|635-4|.

\section{FlOOOPrime}

RECOMMENDED

12. Rastan AJ, Dege A, Mohr M, Doll N, Falk V, Walther T, Mohr FW: Early and late outcomes of 517 consecutive adult patients treated with extracorporeal membrane oxygenation for refractory postcardiotomy cardiogenic shock. J Thorac Cardiovasc Surg 2010, | 39:302-II, 3 | I.el.

FlOOOPrime RECOMMENDED

13. Sayer GT, Baker JN, Parks KA: Heart rescue: the role of mechanical circulatory support in the management of severe refractory cardiogenic shock. Curr Opin Crit Care 2012, I 8:409-I6.

14. Subramaniam K, Boisen M, Shah PR, Ramesh V, Pete A: Mechanical circulatory support for cardiogenic shock. Best Pract Res Clin Anaesthesiol 2012, 26:131-46.

15. Combes A, Bacchetta M, Brodie D, Müller T, Pellegrino V: Extracorporeal membrane oxygenation for respiratory failure in adults. Curr Opin Crit Care 2012, 18:99-104.

16. Gattinoni L, Carlesso E, Langer T: Clinical review: Extracorporeal membrane oxygenation. Crit Care 201 I, I 5:243.

17. MacLaren G: Lessons in advanced extracorporeal life support. Crit Care Med 2012, 40:2729-31.

18. Malagon I, Greenhalgh D: Extracorporeal membrane oxygenation as an alternative to ventilation. Curr Opin Anaesthesiol 2013, 26:47-52.

\section{FlOOOPrime} RECOMMENDED

19. Zwischenberger JB, Lynch JE: Will CESAR answer the adult ECMO debate? Lancet 2009, 374:|307-8.

20. Cook RW, Powel TG, Pharoah POD, Roberton NRC: Mechanical ventilation for the newborn. Lancet 1988, I:I78-9.

21. Mike V, Krauss AN, Ross GS: Neonatal extracorporeal membrane oxygenation (ECMO): clinical trials and the ethics of evidence. J Med Ethics 1993, 19:212-8. 
22. MacLaren G, Moran JL, Chalwin RP, Graham PL: ECMO: expertise and equipoise. Crit Care Resusc 2010, I2:213-5.

23. Extracorporeal Life Support Organization: ECLS Registry Report. International summary. Ann Arbor 2013.

24. MacLaren G, Dodge-Khatami A, Dalton HJ, Adachi I, Almodovar M, Annich G, Bartlett R, Bronicki R, Brown K, Butt W, Cooper D, Demuth M, D'Udekem Y, Fraser C, Guerguerian A, Heard M, Horton S, Ichord R, Jaquiss R, Laussen P, Lequier L, Lou S, Marino B, McMullan M, Ogino M, Peek G, Pretre R, Rodefeld M, Schmidt A, Schwartz S, et al.: Joint statement on mechanical circulatory support in children: a consensus review from the Pediatric Cardiac Intensive Care Society and Extracorporeal Life Support Organization. Pediatr Crit Care Med 2013, I4:SI-2.

25. Peura JL, Colvin-Adams M, Francis GS, Grady KL, Hoffman TM, Jessup M, John R, Kiernan MS, Mitchell JE, O’Connell JB, Pagani FD, Petty M, Ravichandran P, Rogers JG, Semigran MJ, Toole JM: Recommendations for the use of mechanical circulatory support: device strategies and patient selection: a scientific statement from the American Heart Association. Circulation 20I2, I 26:2648-67.

FlOOOPrime RECOMMENDED

26. Noah MA, Peek GJ, Finney SJ, Griffiths MJ, Harrison DA, Grieve R, Sadique MZ, Sekhon JS, McAuley DF, Firmin RK, Harvey C,
Cordingley Jl, Price S, Vuylsteke A, Jenkins DP, Noble DW, Bloomfield R, Walsh TS, Perkins GD, Menon D, Taylor BL, Rowan KM: Referral to an extracorporeal membrane oxygenation center and mortality among patients with severe 2009 influenza A(HINI). JAMA 20II, 306:1659-68.

\section{FlOOOPrime}

RECOMMENDED

27. Pham $T$, Combes A, Rozé $H$, Chevret $S$, Mercat $A$, Roch $A$, Mourvillier B, Ara-Somohano C, Bastien O, Zogheib E, Clavel M, Constan A, Marie Richard J, Brun-Buisson C, Brochard L: Extracorporeal membrane oxygenation for pandemic influenza $A(H I N I)$-induced acute respiratory distress syndrome: a cohort study and propensity-matched analysis. Am J Respir Crit Care Med 2013, 187:276-85

FlOOOPrime

RECOMMENDED

28. Peek G], Mugford M, Tiruvoipati R, Wilson A, Allen E, Thalanany MM, Hibbert CL, Truesdale A, Clemens F, Cooper N, Firmin RK, Elbourne D: Efficacy and economic assessment of conventional ventilatory support versus extracorporeal membrane oxygenation for severe adult respiratory failure (CESAR): a multicentre randomised controlled trial. Lancet 2009, 374:|35|-63.

\section{FlOOOPrime}

RECOMMENDED 